\title{
Z -Shaped Microcatheter Tip Shaping for Embolization of Aneurysms at the Proximal A1 Segment of the Anterior Cerebral Artery: A Technical Note
}

\author{
Ha Young Lee, MD', Jae Sung Ahn, MD², Dae Chul Suh, MD', Deok Hee Lee, MD'1
}

Embolization of an aneurysm at the proximal A1 segment of the anterior cerebral artery (ACA) is technically challenging. We treated four consecutive patients with unruptured aneurysms at the proximal portion of the A1 segment of the ACA. We tested various microcatheter tip shapes before we successfully embolized a lesion with a zigzag-shaped microcatheter tip, which was made by steam shaping. The shape of this microcatheter tip resembled the letter " $Z$ ", had a relatively long distal straight segment and was helpful in prompt catheterization of the aneurysm sac and stable placement of the catheter and delivery of coils.

Key Words : Anterior cerebral artery; Endovascular techniques; Intracranial aneurysms; Catheters

Safe and effective endovascular embolization involves many steps, one of which is the secure and effective insertion of the microcatheter tip into the sac of the target aneurysm. During endovascular treatment of aneurysms, it may be difficult or time-consuming to place the microcatheter tip into an aneurysm sac that is located distally or has complex geometry with respect to the parent artery. One difficult type of aneurysm to treat is an aneurysm at the proximal portion of the A1

'Department of Radiology and Research Institute of Radiology, ${ }^{2}$ Neurosurgery, University of Ulsan College of Medicine, Asan Medical Center, Seoul, Korea

Received February 23, 2011;

accepted after revision June 12, 2011.

Correspondence to: Deok Hee Lee, MD, Department of Radiology and Research Institute of Radiology, University of Ulsan, College of Medicine, Asan Medical Center, 86 Asanbyeongwon-gil, Songpa-gu, Seoul 138-736, Korea.

Tel. 82.2.3010.4366 Fax. 82.2.476.0090

E-mail: dhlee@amc.seoul.kr

This is an Open Access article distributed under the terms of the Creative Commons Attribution Non-Commercial License (http://creativecommons.org/licenses/by-nc/3.0) which permits unrestricted non-commercial use, distribution, and reproduction in any medium, provided the original work is properly cited. segment of the anterior cerebral artery (ACA).

The complicated geometry of an aneurysm formed by the acute angle between the terminal segment of the internal carotid artery (ICA) and the A1 segment [1,2] makes it challenging to insert the microcatheter tip into the aneurysm sac. Even after successful selection of the aneurysm, the microcatheter is prone to kick back due to instability. After much experience, we found that a "Z-shaped" microcatheter shaped tip was successful at coil delivery into the sac without a significant kick-backs. We describe here our microcatheter tip shaping method for proximal ACA A1 segment aneurysms by presenting our experience with several patients.

\section{TECHNICAL NOTES}

\section{Patient 1}

A 59-year-old woman was referred to our neurointerventional service for the embolization of an incidentally-found a right ACA A1 segment aneurysm (Fig. 1). After placing the patient under general anesthesia, we attempted to insert the tip of the microcatheter 
(Excelsior SL10; Target Therapeutics/Boston Scientific, Natick, MA, U.S.A.) into the aneurysm sac. Even using various steam-shaped microcatheter tips, it was very difficult to stably position the tip. After steam-shaping the tip into an "S-shape", we were able to insert the microcatheter tip into the sac. During insertion of the framing coil, however, the tip of the microcatheter was kicked back, and the procedure was aborted after more than 90 minutes.

\section{Patient 2}

A 52-year-old woman with an unruptured lesion was referred to us for treatment. Microcatheter positioning was successful using a steam-shaped microcatheter tip at an obtuse angle. Due to its instability, however, compact packing of the aneurysm was not possible (Fig. 2).

\section{Patient 3}

A 59-year-old man was referred for coil embolization of an incidental lesion. We intentionally steam-shaped the tip of the microcatheter (Excelsior SL10) into a " $Z$ shape" with a straight distal segment longer than that of an "S-shaped" tip (Fig. 3A). We were able to advance the microcatheter tip from the terminal ICA bifurcation into the aneurysm sac without any hindrance by the angle between the ICA and ACA A1 segment (Fig. 3B, C). Complete, stable embolization was achieved until the finishing coil had been completely inserted.
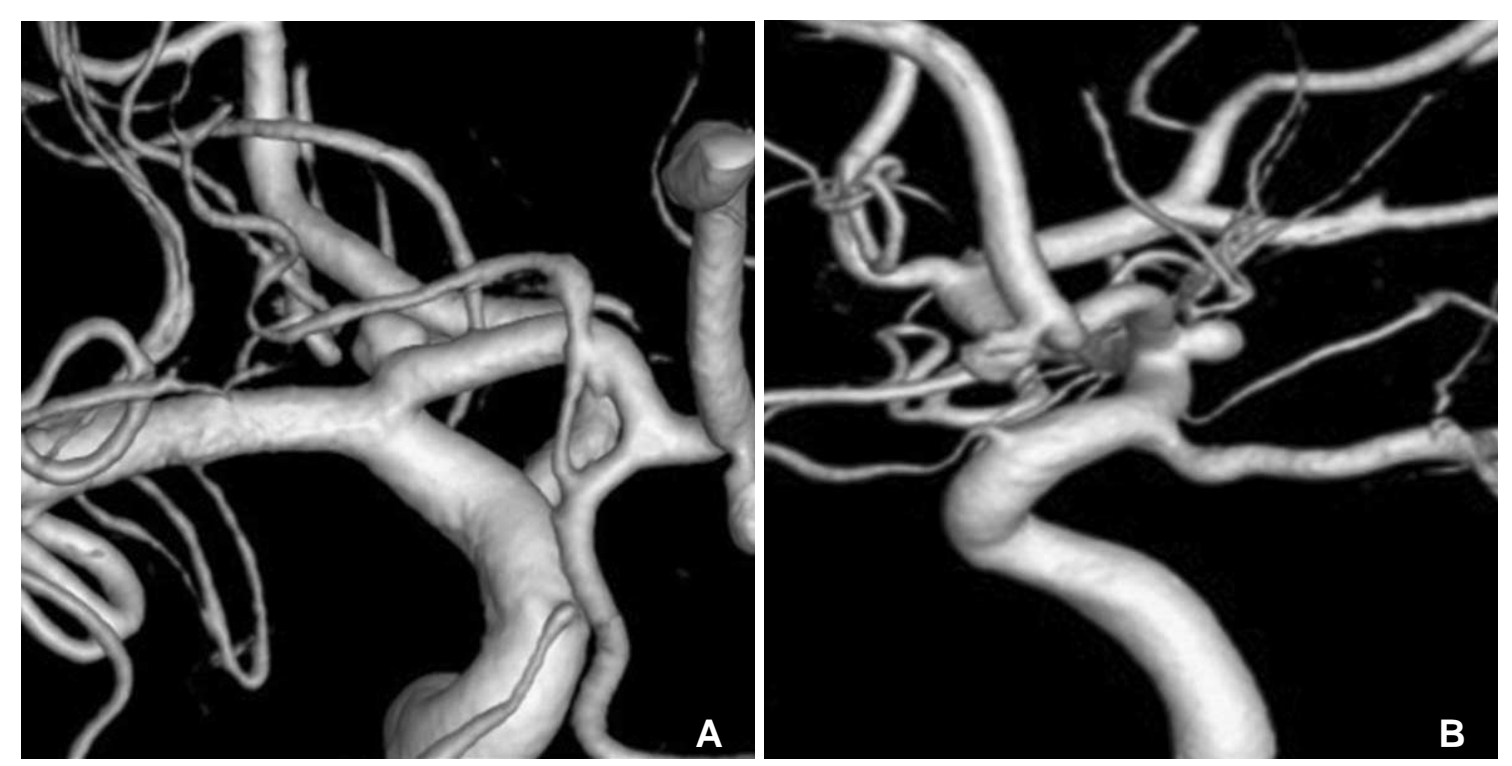

Fig. 1. Case 1, A 59-year-old female.

A, B. Anterior-posterior (AP) and lateral right carotid angiogram with 3D reconstruction demonstrating posteriorly projecting saccular aneurysm arising at right proximal $\mathrm{A} 1$ segment of $\mathrm{ACA}$.

\section{Patient 4}

A 60-year-old man with an incidental lesion was referred for coiling. We tried to use the simple " $\mathrm{Z}$ shaped" microcatheter tip described for Patient 3. However, the initial attempt was unsuccessful because of the rather higher position of the aneurysm sac relative to the main axis of the terminal segment of the

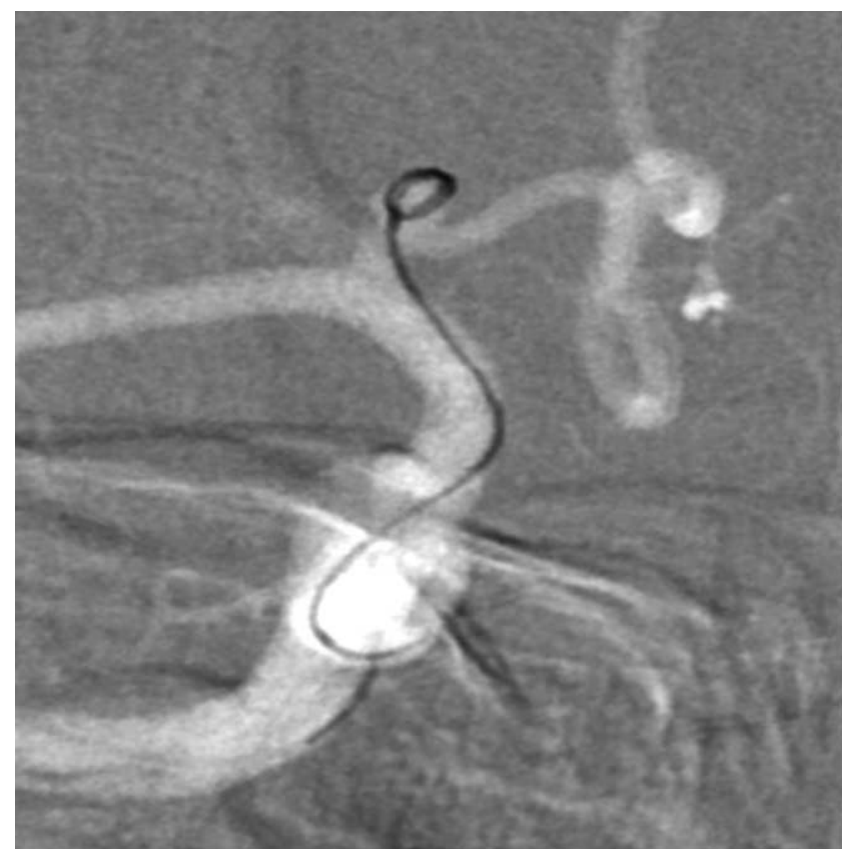

Fig. 2. Case 2, 52-year-old female. Catheterization of aneurysm sac was achieved using a steam-shaped microcatheter in a "Sshape", but compact packing of the aneurysm was not possible due to early kick-back of the microcatheter. 


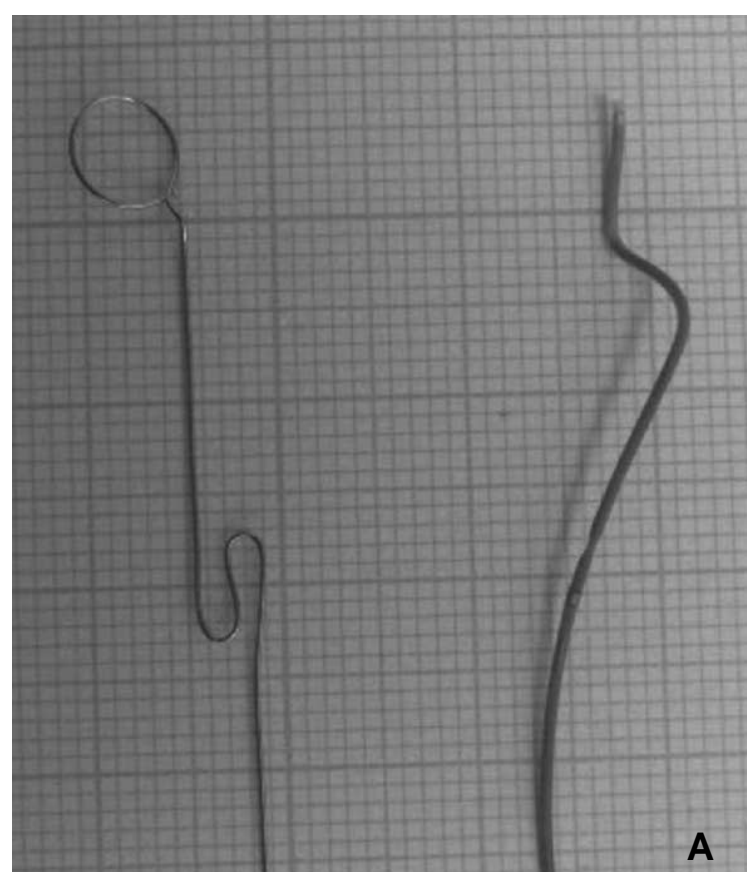

Fig. 3. Case 3, 59-year-old male.

A. The photograph of a steam-shaped distal microcatheter tip in "zigzag-shape" shows more acute angles, and straighter and longer distal segment than usual "S" shape.

B, C. AP and lateral road-map images. Successful catheterization of aneurysm sac was achieved using a microcatheter steamshaped in a "zigzag-shape" and subsequent compact coil packing was followed with stable microcatheter position.
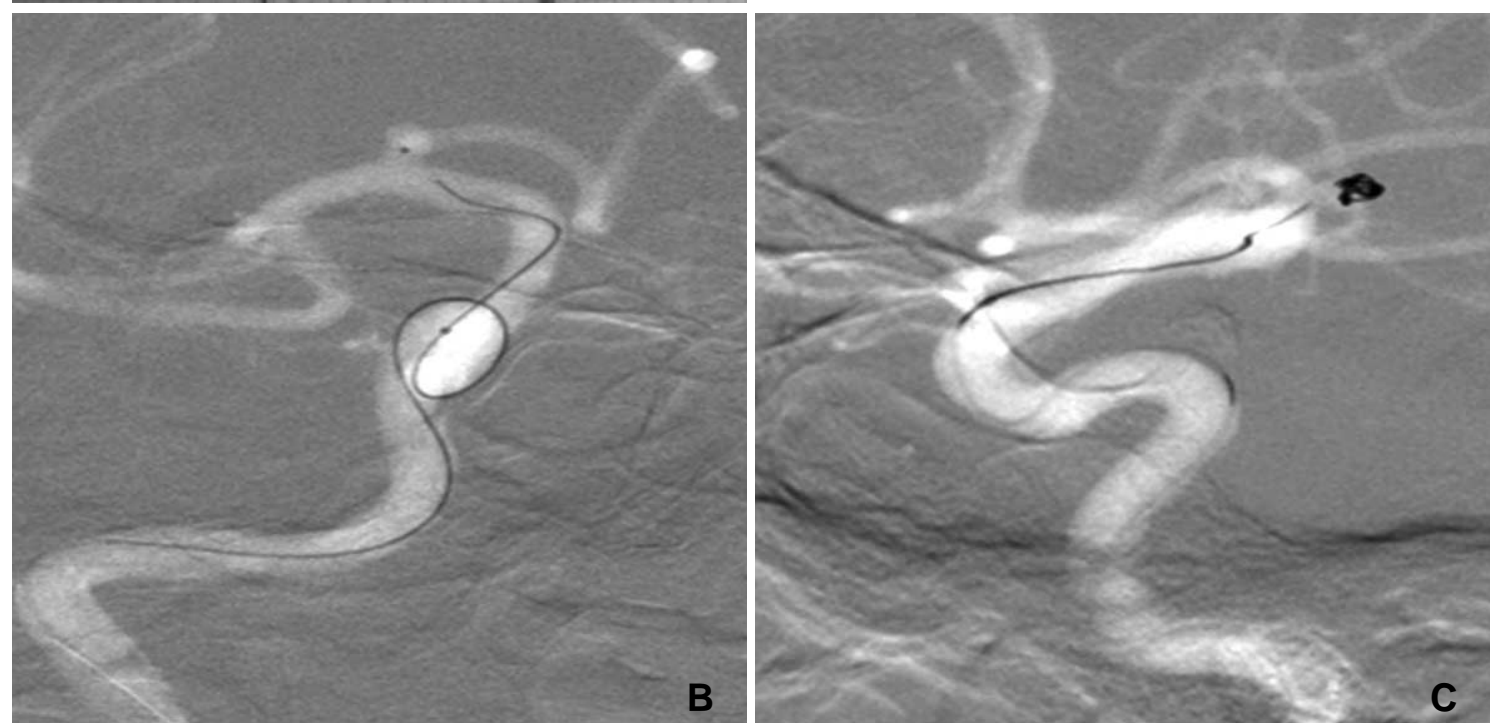

ICA, as shown on a lateral view (Fig. 4A, B). We modified the shape of the tip to correspond to the main direction of the aneurysm sac (Fig. 4C), making compact coil packing possible.

\section{DISCUSSION}

Intra-saccular placement of a microcatheter and stable coil placement are frequently difficult for aneurysms located at the proximal A1 segment of the ACA, as we experienced in Patients 1 and 2 [2]. Our experience with these two patients motivated us to improve our microcatheter navigation and shaping skills. However, there has been little discussion on this

issue [2]. The shapability, durability of the shape, and luminal changes in microcatheters have been evaluated in-vitro, and the adaptability of several microcatheter shapes to paraclinoid aneurysms has been assessed clinically [3, 4].

Several microcatheter shapes adapted to the direction of an aneurysm have been found suitable for paraclinoid aneurysms, include S-shaped, pigtail-shaped, and other shapes [3]. However, shaping a microcatheter tip in accordance with the geometry of the parent artery and aneurysm dome was not successful in Patients 1 and 2. Even after successfully positioning the microcatheter tip, it was not able to insert the coil due to early kick-back of the microcatheter tip. 

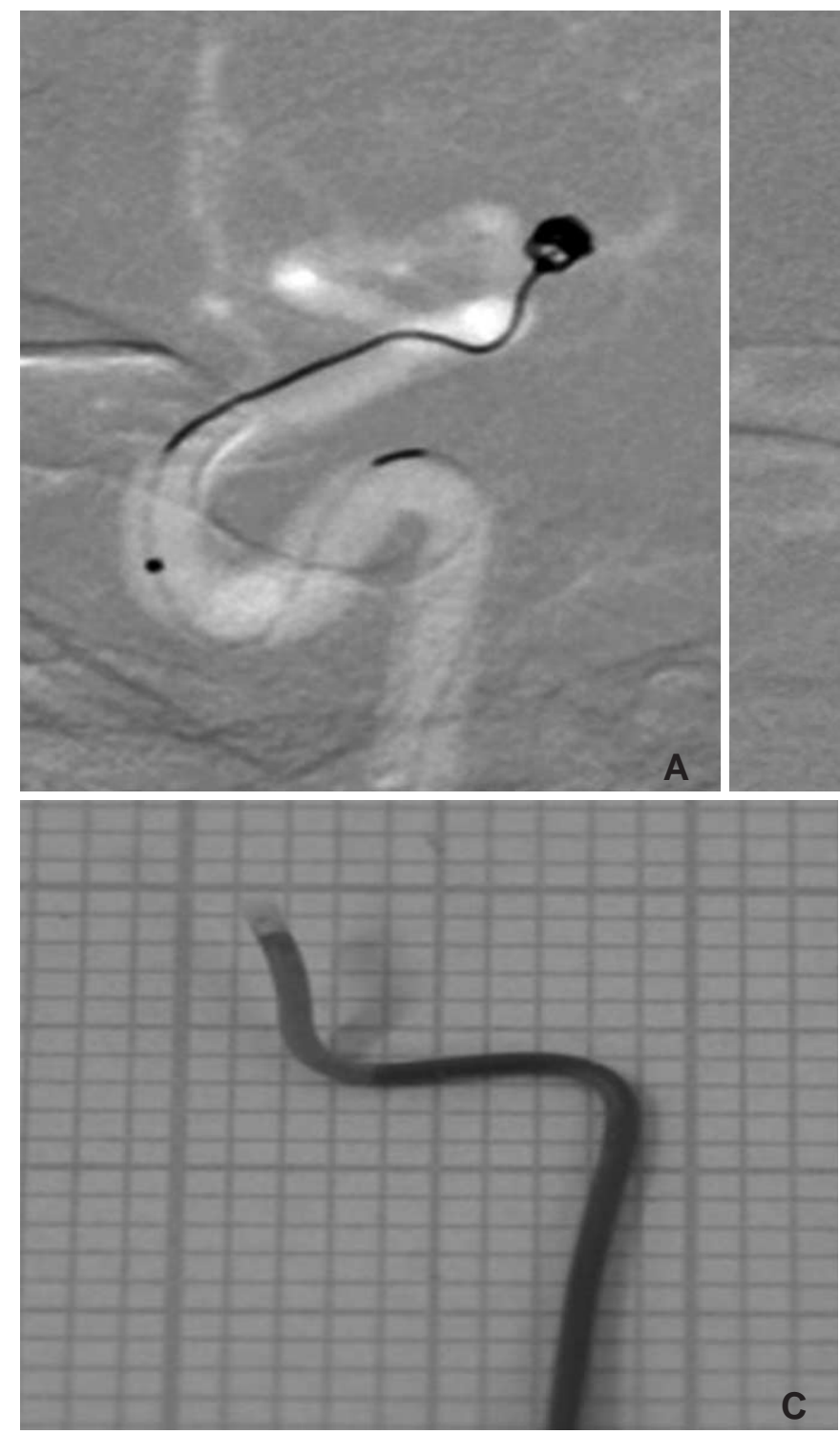

Our experiences with Patients 1 and 2 suggested that a longer distal shaped tip may be more successful. That could be achieved by ignoring the angle between the terminal portion of the ICA and the proximal portion of the A1 segment. We found that the straight part of the microcatheter tip efficiently engaged the sac, while the proximal angles were helpful for the lateral positioning of the distal angle and the supportive action of the more proximal angle. We eventually designed a "Z-shaped" tip, which was more effective when it had a rather longer distal straight segment.

As we showed for Patient 4, the distal straight tip should be shaped according to the direction of the terminal segment of the ICA on lateral projection. If the terminal ICA has a rather steep angle, the tip of the catheter could not be easily inserted into the aneurysm
Fig. 4. Case 4, 60-year-old male.

A, B. AP and lateral road-map images. The aneurysm arises in right proximal $\mathrm{A} 1 \mathrm{ACA}$, in a rather higher position when compared with the main axis of the terminal segment of the ICA on lateral view. Stable coil packing was possible with steam-shaping of the microcatheter in modified "zigzag-shape".

C. We modified the the tip shaping according to the main direction of the aneurysm sac, because the axis of aneurysm was rather higher from the terminal ICA bifurcation in this case.

sac because of the angle. In this situation, a modified $\mathrm{Z}$ shape with a slight superiorly directed tip may be helpful.

In conclusion, we found that a "Z-shaped" microcatheter tip was helpful in achieving effective and secure microcatheter tip positioning for the embolization of proximal ACA A1 segment aneurysms, which are rare but difficult to treat with the endovascular techniques.

\section{References}

1. Rosner SS, Rhoton AL Jr, Ono M, Barry M. Microsurgical anatomy of the anterior perforating arteries. J Neurosurg 1984;61:468-485.

2. Gupta R, Horowitz MB, Gilman S. Neuroform stent-assisted coil embolization of a ruptured A1 segment anterior cerebral artery aneurysm. J Neuroimaging 2006;16:117-119 


\section{Embolization of Aneurysms at the Proximal A1 Segment}

3. Kwon BJ, Im SH, Park JC, Cho YD, Kang HS, Kim JE, et al. Shaping and navigating methods of microcatheters for endovascular treatment of paraclinoid aneurysms. Neurosurgery 2010;67:3440
4. Kiyosue H, Hori Y, Matsumoto S, Okahara M, Tanoue S, Sagara $Y$, et al. Shapability, memory, and luminal changes in microcatheters after steam shaping: a comparison of 11 different microcatheters. AJNR Am J Neuroradiol 2005;26:2610-2616 